Revista Brasileira do Esporte Coletivo - v. 3. n. 1. 2019

\title{
CONFIABILIDADE DE UM MÉTODO SIMPLES PARA MENSURAÇÃO DA VELOCIDADE DA BOLA NO ARREMESSO DO HANDEBOL CONFIABILIDADE NO ARREMESSO DO HANDEBOL
}

${ }^{1}$ João Eduardo Pereira da Silva Junior; ${ }^{2}$ Candida Luiza Tonizza de Carvalho; ${ }^{3}$ Alexandre Gomes de Almeida

\begin{abstract}
RESUMO
Introdução: $O$ arremesso é uma ação técnica importante no handebol, pois é por meio dela que se realiza o gol. Por ser uma ação que na maioria das vezes demanda muita potência, vários métodos para mensurar a velocidade da bola no arremesso podem ser utilizadas. Assim, se fazem necessários estudos de métodos simples para que possam ser alternativas no dia a dia de treinadores. Objetivo: O objetivo desse estudo foi analisar a confiabilidade intra e interavaliadores de um método simples para mensuração da velocidade da bola. Método: Três avaliadores realizaram a mensuração da velocidade da bola de 31 atletas de handebol de areia da categoria juvenil. Foi utilizado o método de análise em duas dimensões para mensurar a velocidade da bola. Para a análise da confiabilidade intra-avaliador foi utilizado o test t pareado. Já para a análise da confiabilidade interavaliador utilizouse a análise de variância (ANOVA) one-way e o teste de post hoc de Scheffe, para determinar as médias significamente diferentes. A análise estatística foi realizada utilizando o software IBMSPSS STATISTICS 20.0, com nível de significância pré-estabelecido em $p<0,05$. Resultados e discussão: Foi encontrado que a diferença entre os resultados encontrados pelos avaliadores 1 e 2 ( $p=0,966), 1$ e $3(p=0,965)$ e 2 e $3(p=1)$ não apresentaram diferença estatisticamente significativa, porém quando foram comparados os resultados de um mesmo avaliador, 0 avaliador $1(p=0,001)$ apresentou diferença estatisticamente significativa, já o avaliador $2(p=0,302)$ e 0 avaliador $3(p=0,305)$ não apresentaram diferença estatisticamente diferentes. Conclusão: $O$ método de análise em duas dimensões apresentou alta confiabilidade. Além de fácil execução e baixo custo, podendo ser uma alternativa no âmbito esportivo no auxílio na prescrição e monitoramento do treinamento.
\end{abstract}

Palavras-chave: Reprodutibilidade, Repetibilidade, Cinemetria, Esportes.

\section{INTRA AND INTER RATER RELIABILITY OF A SIMPLE METHOD FOR BALL VELOCITY MEASUREMENT}

\section{ABSTRACT}

Introduction: The throw is an important technical action in handball, for the reason that it is through it that the goal is accomplished. Because it is an action that often demands a lot of power, several methods to measure the speed of the ball in the throw can be used. Thus, studies of simple methods can be useful on coaches' daily. Objective: The objective of this study was to analyze the intra and interrater reliability of a simple method to measure the velocity of the ball. Method: Three evaluators performed the ball speed measurement of 31 juvenile handball players. The two-dimensional analysis method was used to measure the velocity of the ball. For the analysis of intra-rater reliability, the paired t-test was used. The one-way analysis of variance (ANOVA) and the Scheffe post-hoc test were used to analyze the inter-rater reliability to determine significantly different means. Statistical analysis was performed using the IBMSPSS STATISTICS 20.0 software, with significance level preestablished at $p<0.05$. Results and discussion: It was found that the difference between the results found by the evaluators 1 and $2(p=0.966), 1$ and $3(p=0.965)$ and 2 and $3(p=1)$ did not present a statistically significant difference, however, when the results of the same evaluator were compared, the evaluator $1(p=0.001)$ presented a statistically significant difference, whereas the evaluator $2(p=$ $0.302)$ and the evaluator $3(p=0.305)$ did not present a statistically different difference. Conclusion: The two-dimensional analysis method presented high reliability In addition to easy execution and low cost, it can be an alternative in the sports area in aiding in the prescription and monitoring of the training.

Keywords: Reproducibility, Repeatability, Kinemetry, Sports.

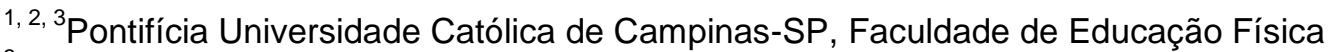

${ }^{3}$ Universidade Estadual de Campinas-SP, Programa de Pós-Graduação em Educação Física 
Endereço: Rua Ângela Gasparine Simioni, $n^{\circ}$ 348. Residencial Fazenda Serrinha - Itatiba, SP

E-mail: joaoeduardojunior@hotmail.com; cltcarvalho2006@puc-campinas.edu.br; agalmeida74@gmail.com

\section{INTRODUÇÃO}

No handebol, o arremesso é a ação técnica de maior importância, pois é por meio dele que se atinge o objetivo do jogo, que é marcar mais gols que 0 adversário. A execução do arremesso varia de acordo com as situações do jogo, podendo ser realizada com os pés no solo (arremesso com apoio) ou no caso do arremesso em suspensão (saltando) (GRECO; ROMERO, 2012). Por ser uma ação que imprime grande velocidade à bola, pode-se dizer que ela é realizada com uma grande potência (SOUZA et al., 2006). Comparando os dois tipos de arremesso, a velocidade da bola do arremesso apoiado é superior ao arremesso em suspensão, principalmente porque as técnicas possuem diferenças na velocidade máxima de flexão, rotação e rotação interna do ombro (WAGNER et al., 2011).

A velocidade de saída da bola pode atingir valores superiores a $21 \mathrm{~m} / \mathrm{s}$, o que reduz as possibilidades de defesa do goleiro (MONTILLA; VINUESA; SICILIA, 2001). Bayios e Boudolos (1982) demonstraram a importância da velocidade da bola quando afirmaram que a combinação dela com a melhor acurácia contribuem para um melhor desempenho do jogador de handebol na partida. No entanto, os níveis de oposição ao arremessador influenciam a velocidade, ou seja, diminuindo à medida que se aumenta o número de opositores (RIVILLA-GARCIA et al., 2011). Por isso, em jogos oficiais os arremessos convertidos em gols não apresentam necessariamente velocidades da bola superiores aos que não foram gol (CORTÉS et al., 2017a). Diante disso, o monitoramento das variações da velocidade da bola em treinamentos e competições pode fornecer informações importantes para a preparação de equipes de handebol.

Existem vários métodos para mensuração da velocidade da bola, como por exemplo, o utilizado por Rousanoglou et al., (2014), em que utilizaram um dispositivo emissor de feixe de laser e um sistema eletrônico detectores infravermelhos de feixe de laser. Outro método é por meio da utilização de um radar gun, que envia um sinal de rádio frequência, em seguida, recebe 0 mesmo sinal de volta depois que ele é refletido pelo objeto alvo (efeito Doppler) (CORTÉS et al., 2017b). Pode ser utilizado também a cinemetria, que consiste em um método que se realiza a filmagem do gesto esportivo, por meio de câmera digital e as imagens coletadas são analisadas em um software (AMADIO et al., 2016). Este método pode ser realizado através de uma análise bidimensional (2D), em que se utiliza o sistema de coordenadas $x$ e $y$ para medir o deslocamento da bola (JÖRIS et al., 1985) ou de uma análise tridimensional (3D), utilizando duas ou mais câmeras de vídeo para determinar as coordenadas $\mathrm{x}$, y e $\mathrm{z}$ (MONTES et al., 2012).

Embora a análise 3D seja um método consagrado, a sua utilização no cotidiano se torna inviável devido a sua complexidade, demanda de tempo e alto custo. Por isso, métodos mais práticos e de baixo custo têm sido estudados, como por exemplo, a pesquisa de Balsalobre-Fernández, Glaister e Lockey (2015) que validou um aplicativo para mensurar a altura do salto a partir de vídeos obtidos em aparelhos de celular ou tablets. Portanto, para medir a velocidade da bola é importante que métodos simples também sejam desenvolvidos para que possam ser alternativas no dia a dia de treinadores. Assim, o objetivo deste estudo consistiu em analisar a confiabilidade intra e interavaliadores da análise $2 \mathrm{D}$, para mensuração da velocidade da bola em atletas de handebol de areia.

\section{MÉTODO}

Três voluntários foram selecionados como avaliadores, sendo dois acadêmicos e um graduado em Educação Física. O estudo foi conduzido em forma de teste-reteste (HOPKINS, 2000), onde no primeiro dia cada avaliador realizou mensurações de velocidade da bola e, após sete dias (FERREIRA et al., 2010), repetiram esses procedimentos. Os dados obtidos no primeiro dia foram registrados e armazenados de modo que os avaliadores não tivessem acesso aos dados uns dos outros até o último dia de análise.

\section{Obtenção das Imagens dos Arremessos \\ As imagens utilizadas para} mensurações da velocidade da bola foram obtidas a partir dos arremessos de atletas da Seleção Brasileira Juvenil de Handebol de Areia, sendo 15 atletas do sexo masculino e 16 atletas do sexo feminino, com média de idade de $17 \pm 1$ anos, que participavam da fase de treinamento na cidade de Mogi MirimSP, promovida pela Confederação Brasileira de Handebol. 
Para a realização do teste, foi solicitado que os atletas realizassem dois arremessos, sendo executados na linha dos seis metros. A orientação para os arremessos é de que realizassem tentando aplicar a maior velocidade possível à bola. Antes da execução dos arremessos foi realizado um aquecimento específico com duração de cinco minutos, contendo exercícios coordenativos.
Para registrar as imagens foi utilizada uma câmera digital (Sony HDR-CX220), fixada em um tripé, que enquadrou todo o ambiente da coleta. A câmera foi posicionada no plano sagital a uma distância de seis metros dos atletas e a uma distância de quatro metros da linha de fundo (Figura 1).

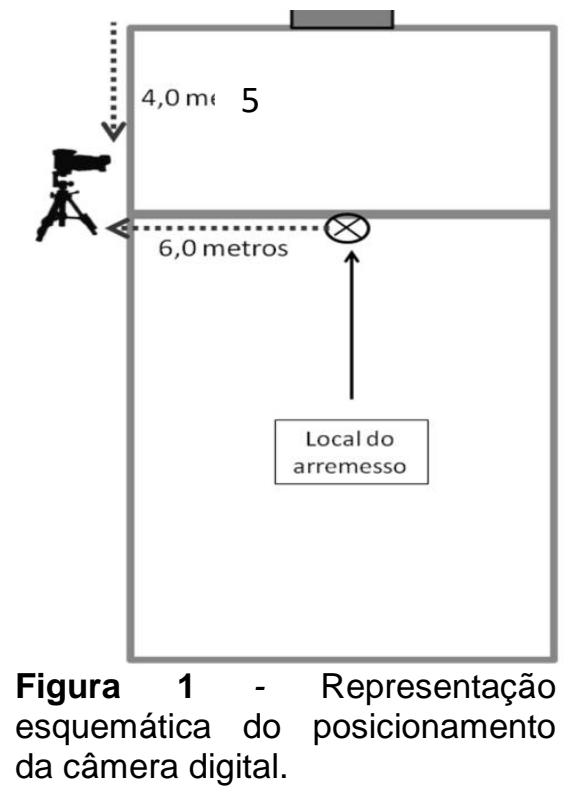

Na calibração foram utilizados marcadores espaciais posicionados na quadra em que a distância entre os marcadores foi de 150 centímetros (Figura 2).

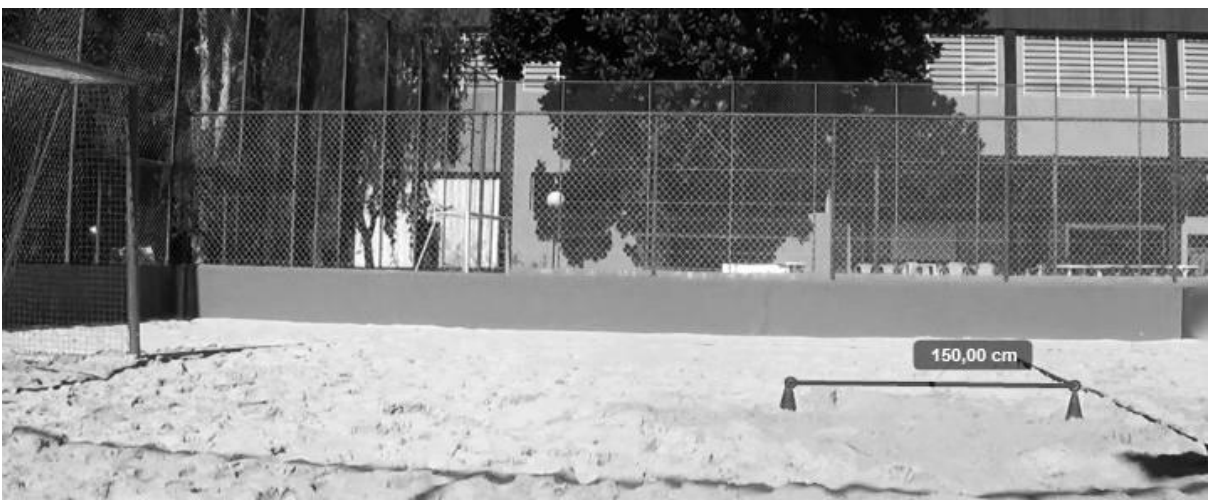

Figura 2 - Representação da calibração do ambiente.

\section{Mensuração da Velocidade da Bola}

Antes de realizar as análises, todos avaliadores foram submetidos a um treinamento que consistiu em explicações sobre o protocolo e por um período de familiarização, onde realizaram a mensuração da velocidade da bola de quinze arremessos, que correspondeu a pelo menos $10 \%$ do total da amostra (TABACHNICK, FIDELL, 2007).

Tanto no teste como no reteste, cada avaliador mediu a velocidade da bola de 124 arremessos que foram analisados em computadores com tela de no mínimo 15 polegadas, utilizando um mouse. Foi utilizado o software Kinovea (versão 0.8.15; licença gratuita), sendo realizado 0 processo de desentrelaçamento das imagens $e$, posteriormente, determinadas as coordenadas (x1 e y1) no primeiro quadro, estabelecido pelo instante após a saída da bola da mão do atleta (Figura 3A), e nos dois quadros subsequentes foram determinadas as coordenadas (x2 e y2) (Figura 3B). 


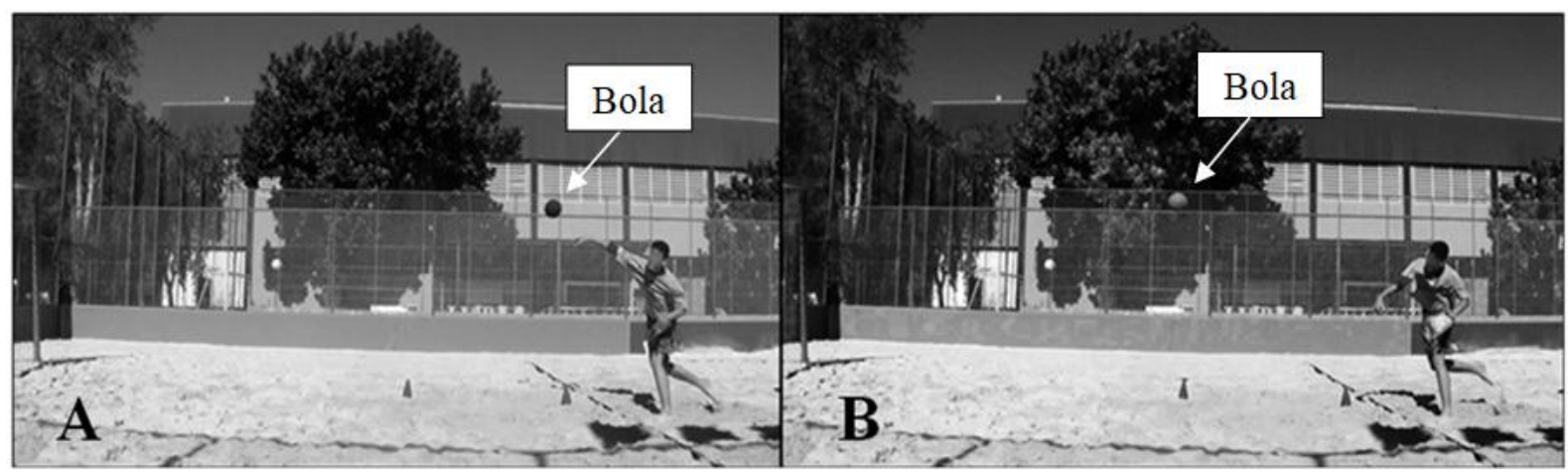

Figura 3 - Instante da saída da bola da mão do atleta (A); Dois quadros após a saída bola (B).

Para calcular a velocidade da bola, primeiro foi estabelecida a diferença entre as coordenadas ( $\mathrm{x} 1$ e $\mathrm{x} 2)$ e as coordenadas ( $\mathrm{y} 1$ e y2). Para a obtenção do valor de deslocamento da bola (d) foi utilizado o teorema de Pitágoras, onde o valor obtido foi dividido por cem para ser expresso em metros.

onde:

$$
d=\left(\sqrt{ } x^{2}+y^{2}\right) / 100
$$

$x=a$ diferença entre as coordenadas $x 1$ e $x 2$; e y1 e y2.

$$
y=a \text { diferença entre as coordenadas }
$$

$\mathrm{O}$ valor obtido foi dividido pelo tempo $(\mathrm{t}) \mathrm{de}$ 0,067 s (obtido através do número de quadros por segundo, sendo adotados trinta quadros por segundo), assim foi possível utilizar a fórmula da velocidade para mensurar a velocidade de saída da bola $(\mathrm{Vb})$.

$$
\mathrm{Vb}=\mathrm{d} / \mathrm{t}
$$

em que:

$\mathrm{d}=$ deslocamento da bola;

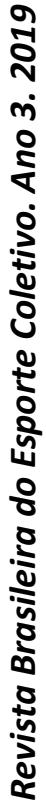

\section{Análise Estatística}

Os resultados da mensuração da velocidade da bola foram analisados de forma exploratória, onde foi analisado a média, desvio padrão, maior e menor realizados nos dias de análise pelos avaliadores. $\mathrm{Na}$ análise estatística, foi possível determinar a significância entre as medidas do mesmo avaliador (confiabilidade intra-avaliador) e de avaliadores diferentes (confiabilidade interavaliador).

A confiabilidade intra-avaliador, ou seja, a habilidade de um avaliador em dar o mesmo resultado em diferentes momentos, foi avaliada pela diferença entre as medidas do par (mesmo avaliador em dois momentos distintos) utilizando o test t pareado.

A confiabilidade interavaliador, ou seja, o fato da velocidade da bola dar o mesmo resultado entre avaliadores diferentes foi realizada por meio da análise de variância (ANOVA) one-way e o teste de post hoc de Scheffe, para determinar as médias significamente diferentes. A análise estatística foi realizada utilizando o software Statistical Package for the Social Sciences (SPSS, versão 20.0), com nível de significância préestabelecido em $p<0,05$. 
RESULTADOS

Com 0 intuito de analisar a confiabilidade do método, foi realizada a média dos resultados obtidos pelos avaliadores no teste e no reteste.

Tabela 1 - Média, desvio padrão, maior e menor das velocidades de saída da bola $(\mathrm{m} / \mathrm{s})$ dos atletas.

\begin{tabular}{ccccccc}
\hline & \multicolumn{2}{c}{ Avaliador 1 } & \multicolumn{2}{c}{ Avaliador 2 } & \multicolumn{2}{c}{ Avaliador 3 } \\
\cline { 2 - 7 } & Teste & Reteste & Teste & Reteste & Teste & Reteste \\
\hline Média & 20,72 & 20,81 & 20,61 & 20,56 & 20,61 & 20,67 \\
Desvio Padrão & $\pm 1,59$ & $\pm 1,61$ & $\pm 1,63$ & $\pm 1,69$ & $\pm 1,70$ & $\pm 1,57$ \\
Maior & 25,40 & 25,57 & 25,27 & 25,55 & 25,72 & 25,17 \\
Menor & 18,10 & 18,04 & 17,49 & 17,41 & 17,98 & 18,03 \\
\hline
\end{tabular}

Os resultados apresentados na tabela 1 demonstram que as médias das velocidades de saída bola mensuradas pelos avaliadores no teste e no reteste se mantém próximas. Apresentando também como a maior velocidade de saída da bola o valor de 25,72 $(\mathrm{m} / \mathrm{s})$ mensurada pelo avaliador 3 e a menor velocidade obtida foi de $17,41 \quad(\mathrm{~m} / \mathrm{s})$, determinada pelo avaliador 2 .
Quando comparados os resultados do teste e do reteste, verifica-se que os avaliadores 1 e 3 , apresentaram um aumento da média das velocidades no reteste em comparação ao teste, no entanto o avaliador 2 apresentou uma diminuição da média das velocidades no reteste em comparação ao teste.

Tabela 2 - Resultado para confiabilidade intra e interavaliador.

\begin{tabular}{cccc}
\hline Intra-avaliador & $p$ & Interavaliadores & $p$ \\
\hline Av1 & $0,001^{*}$ & Av1 x Av2 & 0,966 \\
Av2 & 0,302 & Av1 x Av3 & 0,965 \\
Av3 & 0,305 & Av2 x Av3 & 1 \\
\hline $\begin{array}{l}\text { Legenda: Av1 = Avaliador 1; Av2 = Avaliador 2; Av3 = Avaliador 3; } \\
\text { = significância; }{ }^{*}=\text { diferença estatisticamente significativa. }\end{array}$
\end{tabular}

$\mathrm{Na}$ tabela 2 são apresentados os dados de confiabilidade quando comparadas as médias dos avaliadores. $\mathrm{Na}$ avaliação da confiabilidade intra-avaliador, por um mesmo avaliador em dias diferentes, observa-se que apenas os resultados obtidos pelo avaliador 1 , possui diferença estatisticamente significativa, enquanto os avaliadores 2 e 3 não apresentaram diferença estatisticamente significativa.

Em relação à confiabilidade interavaliador, não houve diferença estatisticamente significativa entre as medidas dos avaliadores, demonstrando assim, um método com uma boa confiabilidade interavaliadores.

\section{DISCUSSÃO}

Não foram encontrados estudos em que foi realizada a velocidade da bola no handebol de areia, no entanto os resultados encontrados nesse estudo foram confrontados com resultados de estudos em atletas de handebol de quadra.

Foi encontrado neste estudo a maior velocidade da bola de $25,72 \mathrm{~m} / \mathrm{s}$ determinado pelo avaliador 3 , a menor velocidade da bola de $17,41 \mathrm{~m} / \mathrm{s}$ determinado pelo avaliador 2 e a maior média foi de $20,81 \mathrm{~m} / \mathrm{s}$ encontrada pelo avaliador 1. Assim, quando comparados aos resultados encontrados por Bayios e Boudolos (1982), a média encontrada no presente estudo se mostrou menor, onde eles encontraram a média das velocidades de 
$23,51 \mathrm{~m} / \mathrm{s}$ no arremesso com apoio. Entretanto, quando confrontado com o estudo de Rousanoglou et al., (2014), foi encontrado em arremessos em suspensão o valor médio de $17,5 \mathrm{~m} / \mathrm{s}$ e em arremessos com as três passadas foi encontrado valor médio de 19,6 $\mathrm{m} / \mathrm{s}$ para a velocidade da bola. Ambos os estudos utilizaram o método do feixe de laser para determinar a velocidade da bola e os participantes dessas pesquisas foram atletas do sexo masculino com média de idade de 25 e 22 anos.

Em estudos onde foi mensurada a velocidade da bola durante as partidas do Campeonato Mundial de Handebol Masculino de 2013 (CORTÉS et al., 2017a; CORTÉS et al., 2017b), por meio de um radar gun, foram encontradas as médias da velocidade da bola de $22,11 \mathrm{~m} / \mathrm{s}$ e também para cada local do gol, onde foi encontrado a média da velocidade da bola no lado direito de $22,44 \mathrm{~m} / \mathrm{s}$, no centro de $23,93 \mathrm{~m} / \mathrm{s}$ e do lado esquerdo de $21,86 \mathrm{~m} / \mathrm{s}$, apresentando assim, uma média maior a média encontrada nesse estudo.

Em outro estudo no qual foi utilizado o método do radar gun, com atletas do sexo masculino e média de idade de 22 anos, foi encontrada a média de velocidade da bola de 23,98 m/s com a realização das três passadas (MARQUES et al., 2007), apresentando assim uma média maior a encontrada nesse estudo. Ainda com a utilização desse mesmo método, foi realizado uma pesquisa, com indivíduos do sexo masculino com média de 21 anos de idade e do sexo feminino com média de idade de 21 anos em que realizaram o arremesso em uma primeira sessão os atletas do sexo masculino apresentaram a velocidade da bola com o menor valor de $74,2 \mathrm{~km} / \mathrm{h}(20,61 \mathrm{~m} / \mathrm{s})$ e maior valor de $105 \mathrm{~km} / \mathrm{h}(29,17 \mathrm{~m} / \mathrm{s})$ e as atletas do sexo feminino foram encontrados 0 menor valor de $48,8 \mathrm{~km} / \mathrm{h}(13,55 \mathrm{~m} / \mathrm{s})$ e 0 maior valor de $78 \mathrm{~km} / \mathrm{h}(21,67)$, após os arremessos foi dado aos indivíduos um feedback a respeito dos arremessos. A velocidade da bola foi novamente mensurada em outra sessão, encontrando o menor valor de $79 \mathrm{~km} / \mathrm{h}(21,94 \mathrm{~m} / \mathrm{s})$ e valor máximo de 108 $\mathrm{km} / \mathrm{h}(30 \mathrm{~m} / \mathrm{s})$ para atletas do sexo masculino e de mínimo $50,8 \mathrm{~km} / \mathrm{h}(14,11 \mathrm{~m} / \mathrm{s})$ e máximo de $81,1 \mathrm{~km} / \mathrm{h}(22,53 \mathrm{~m} / \mathrm{s})$ para atletas do sexo feminino (ŠTIRN et al., 2017). Apresentando resultados menores aos encontrados no presente estudo pelos atletas do sexo masculino e resultados maiores aos mensurados nesse estudo pelas atletas do sexo feminino.

No estudo de Saavedra et al., (2018), onde foi realizado 0 arremesso dos sete metros sem oposição, com a utilização de radar gun, com atletas do sexo feminino com média de idade de 24 anos, foi encontrada a média da velocidade da bola em 74,8 km/h $(20,77 \mathrm{~m} / \mathrm{s})$. Com atletas com média de 18 anos de idade foi encontrada a velocidade da bola de $73,5 \mathrm{~km} / \mathrm{h}(20,42 \mathrm{~m} / \mathrm{s})$. Com atletas com média de idade de 16 anos encontrou $67,8 \mathrm{~km} / \mathrm{h}(18,83 \mathrm{~m} / \mathrm{s})$. Com atletas de 14 anos de média de idade, foi encontrado $68,4 \mathrm{~km} / \mathrm{h}$ $(19 \mathrm{~m} / \mathrm{s})$ para a velocidade da bola. Quando comparados à média da velocidade das atletas com média de 24 anos, os resultados encontrados nesse estudo apresentam valores similares. Porém em relação às outras idades, o presente estudou apresentou valores superiores.

Em um estudo realizado por Serrien et al., (2016), com atletas do sexo masculino com média de idade de 18 anos, foi realizada a mensuração da velocidade da bola no arremesso dos sete metros em alguns meses, por meio da utilização da cinemetria da análise em três dimensões. Encontrou no mês de fevereiro a maior velocidade de $23,26 \mathrm{~m} / \mathrm{s}$ e a menor de $17,18 \mathrm{~m} / \mathrm{s}$, no mês de abril encontrou a maior em $23,83 \mathrm{~m} / \mathrm{s}$ e a menor em $16,46 \mathrm{~m} / \mathrm{s}$ e no mês de maio a maior velocidade encontrada foi de 22,81 e a menor $15,44 \mathrm{~m} / \mathrm{s}$, apresentando resultados menores aos encontrados nesse estudo. Ainda, em outro estudo que também foi utilizado o método de três dimensões, porém com a realização das três passadas, e com atletas com média de idade de 21 anos, foi encontrado valor da média das velocidades da bola em 21,82 m/s (AKL, SALEM, 2018), apresentando uma média maior ao do presente estudo.

Em um estudo onde foi utilizado o método de análise em duas dimensões com atletas do sexo masculino de 23 anos de média de idade, foi realizado o arremesso com oposição, onde foi encontrado valor médio da velocidade da bola de 20,54 m/s (MONTILLA; VINUESA, SICILIA, 2001), o qual apresentou valor menor ao quando comparado com esse estudo. Entretanto, no estudo realizado por Wagner, Fuchs e Von Duvillard (2017), onde também foi utilizado o método de análise em duas dimensões, com indivíduos do sexo masculino, divididos em elite, sub-elite e não elite. No qual os indivíduos do grupo elite com média de idade de 25 anos, foi encontrado média da velocidade da bola em $25,7 \mathrm{~m} / \mathrm{s}$, no grupo sub-elite com média de idade de 23 anos, foi encontrado valor para a velocidade da bola em 23,5 m/s e no grupo de não elite com média de idade de 26 anos, foi encontrado velocidade da bola em $22,2 \mathrm{~m} / \mathrm{s}$, todos os grupos, apresentaram maiores 
valores quando comparados aos desse estudo.

O que também aconteceu com o estudo de Hermassi et al., (2018), onde foi mensurada a velocidade da bola em dois momentos com a utilização da análise em duas dimensões no arremesso dos sete metros. Foi encontrado no primeiro momento o valor médio de $31 \mathrm{~m} / \mathrm{s}$ e no segundo momento o valor médio de $30,6 \mathrm{~m} / \mathrm{s}$, valores maiores aos encontrados nesse estudo, porém participaram do estudo atletas com média de idade de 20 anos. Quando comparada a outro estudo que também utilizou o método em duas dimensões para mensurar a velocidade da bola foi encontrado valor médio de 17,2 m/s em atletas com média de idade de 22 anos (JÖRIS et al., 1985).

Dessa forma, para um protocolo ser considerado confiável é necessário que se obtenha sempre o mesmo resultado em

\section{CONCLUSÃO}

A mensuração da velocidade da bola através da análise $2 \mathrm{D}$ em software gratuito demonstrou alta confiabilidade intra e interavaliadores. Além disso, a fácil execução e o baixo custo dos procedimentos apontam para uma ampla utilização no âmbito esportivo, subsidiando a prescrição e monitoramento do treinamento. Assim, estudos com outras modalidades esportivas e ações técnicas devem ser conduzidos para aumentar 0 conhecimento nessa área. medidas repetidas da mesma variável. Assim, a confiabilidade está associada à precisão do método, o qual deve possuir o mínimo de erro sistemático ou aleatório. Onde algumas das causas desses erros, ocorrem devido à variação dos avaliadores, das ferramentas de medida e o que está sendo mensurado (PRETTY, ADDY, MAUPOMÉ, 2004).

Diante disso, em diversos estudos foram analisadas a confiabilidade intra e interavalidores, como no estudo de Leão, Viana e Sougey (2017) e no estudo de Carvalho, Mazzer e Barbieri (2012), porém não foram encontrados estudos nos quais foram analisadas a confiabilidade de métodos para mensuração da velocidade da bola. Nos estudos de Leão Viana e Sougey (2017) e de Carvalho, Mazzer e Barbieri (2012), foram encontrados uma boa confiabilidade intra e interavaliadores, assim como também foi encontrado no presente estudo.

\section{REFERÊNCIAS}

AKL, A. R. I.; SALEM, M. H. Effects of center of mass kinematics on ball velocity during jump throwing in handball. Moj Applied Bionics and Biomechanics, v. 2, n. 3, p. 219-221, 2018.

AMADIO, A. C. et al. Métodos de medição em biomecânica do esporte: Descrição de protocolos para aplicação nos centros de excelência esportiva (Rede CENESP-MET). p. 1-23, 2016.

BALSALOBRE-FERNÁNDEZ, C.; GLAISTER, M.; LOCKEY, R. A. The validity and reliability of an iPhone app for measuring vertical jump performance. Journal of Sports Sciences, v. 33, n. 15, p. 1574-1579, 2015.

BAYIOS, I.; BOUDOLOS, K. Accuracy and throwing velocity in handball. Igaku Toshokan, v. 29, n. 2 , p. $186-188,1982$.

CARVALHO, R. M. F. DE; MAZZER, N.; BARBIERI, C. H. Análise da confiabilidade e reprodutibilidade da goniometria em relação à fotogrametria na mão. Acta Ortopédica Brasileira, v. 20, n. 3, p. 139-149, 2012.

CORTÉS, J. C. Z. et al. Difference of the speed of handball throwing during the competition in relation to efficiency: Analysis between the first and the second half. Journal of Human Sport and Exercise, v. 12, n. Proc3, p. S872-S881, 2017a.

CORTÉS, J. C. Z. et al. Analysis of the throwing speed in the different positions in the field during the competition. Journal of Human Sport and Exercise, v. 12, n. Proc3, p. S882-S891, 2017b.

FERREIRA, E. A. G. et al. Postural assessment software (PAS/SAPO): Validation and reliabiliy. Clinical Science, v. 65, n. 7, p. 675-681, 2010.

GRECO, P. J.; ROMERO, J. J. F. Manual de handebol: Da iniciação ao alto nível. Phorte, 2012.

HERMASSI, S. et al. Relationships between olympic weightlifting exercises, peak power of the upper and lower limb, muscle volume and throwing ball velocity in elite male handball players. Thieme Medical Publishers, p. 1-9, 2018. 
HOPKINS, W. G. Mesaures of reliability in sports medicine and science. Sports Medicine, v. 30, n. 1, p. $1-15,2000$.

JÖRIS, H. J. J. et al. Force, velocity and energy flow during the overarm throw in female handball players. Journal of Biomechanics, v. 18, n. 6, p. 409-414, 1985.

LEÂO, I. C. S.; VIANA, M. T.; SOUGEY, E. B. Reprodutibilidade de respostas na validação de um protocolo do nível de conhecimento tático declarativo no handebol. Revista Brasileira do Esporte Coletivo, v. 1, n. 1, p. 4-7, 2017.

MARQUES, M. C. et al. Relationship between throwing velocity, muscle power, and bar velocity during bench press in elite handball players. International Journal of Sports Physiology and Performance, v. 2, p. 414-422, 2007.

MONTES, F. A. et al. Análise tridimensional do arremesso com apoio no handebol. UNOPAR Científica Ciências Biológicas e da Saúde, v. 14, n. 1, p. 5-8, 2012.

MONTILLA, J. P.; VINUESA, A. S.; SICILIA, A. O. Importancia de la velocidad de salida del balón y de la precisión como parámetros de eficacia en el lanzamiento en salto a distancia en balonmano. Apunts. Educacion Fisica y Deportes, v. 66, p. 44-51, 2001.

PRETTY, I. A.; ADDY, L.; MAUPOMÉ, G. A closer look at diagnosis in clinical dental practice: Part 6. Emerging technologies for detection and diagnosis of noncaries dental problems. Journal of the Canadian Dental Association, 2004.

RIVILLA-GARCIA, J. et al. Influence of opposition on ball velocity in the handball jump throw. Journal of Sports Science and Medicine, v. 10, n. 3, p. 534-539, 2011.

ROUSANOGLOU, E. et al. Ground reaction forces and throwing performance in elite and novice players in two types of handball shot. Journal of Human Kinetics, v. 40, n. 40, p. 49-55, 2014.

SAAVEDRA, J. M. et al. Anthropometric characteristics, physical fitness, and throwing velocity in elite women's handball teams. Journal of Strength and Conditioning Research, v. 32, p. 2294-2301, 2018.

SERRIEN, B. et al. Intra-seasonal variability of ball speed and coordination of two team-handball throwing techniques in elite male adolescent players. International Journal of Computer Science in Sport, v. 15, n. 1, p. 1-21, 2016.

SOUZA, J. DE et al. Alterações em variáveis motoras e metabólicas induzidas pelo treinamento durante um macrociclo em jogadores de handebol. Revista Brasileira de Medicina do Esporte, v. 12, p. 129-134, 2006.

ŠTIRN, I. et al. Frequent immediate knowledge of results enhances the increase of throwing velocity in overarm handball performance. Journal of Human Kinetics, v. 56, n. 1, p. 197-205, 2017.

TABACHNICK, B. G.; FIDELL, L. S. Using multivariate statistics. $2^{\mathrm{a}}$ ed. Harper Collins Publishers, 2007.

WAGNER, $\mathrm{H}$. et al. Performance and kinematics of various throwing techniques in team-handball. Journal of Sports Science and Medicine, v. 10, p. 73-80, 2011.

WAGNER, H.; FUCHS, P. X.; VON DUVILLARD, S. P. Specific physiological and biomechanical performance in elite, sub-elite and in non-elite male team handball players. The Journal of sports medicine and physical fitness, v. 58, n. 1-2, p. 73-81, 2017. 